\title{
Differential Representations of Dynamical Oscillator Symmetries in Discrete Hilbert Space
}

\author{
ANDREAS RUFFING* \\ Zentrum Mathematik, Technische Universität München, Arcisstrasse, 21/H4, D-80333 München, Germany
}

(Received 10 January 2000)

\begin{abstract}
As a very important example for dynamical symmetries in the context of $q$-generalized quantum mechanics the algebra $a a^{\dagger}-q^{-2} a^{\dagger} a=1$ is investigated. It represents the oscillator symmetry $S U_{q}(1,1)$ and is regarded as a commutation phenomenon of the $q$-Heisenberg algebra which provides a discrete spectrum of momentum and space, i.e., a discrete Hilbert space structure. Generalized $q$-Hermite functions and systems of creation and annihilation operators are derived. The classical limit $q \rightarrow 1$ is investigated. Finally the $S U_{q}(1,1)$ algebra is represented by the dynamical variables of the $q$-Heisenberg algebra.
\end{abstract}

Keywords: Quantum mechanics; Harmonic q-oscillators

\section{INTRODUCTION}

The dynamical symmetry $S U(1,1)$ plays an important role in the abstract description of quantum mechanical harmonic oscillators. Recently several approaches to quantum mechanics in discrete space-momentum structures have been given in the context of quantum groups $[15,16,10]$. Others consider $q$-incertainty phenomena [14]. We follow the basic ideas of [10] where the $q$-deformed Heisenberg algebra and its representations are introduced. Our aim is to represent the corresponding algebra of $S U_{q}(1,1)$ symmetry $[1,2]$

$$
a a^{\dagger}-q^{-2} a^{\dagger} a=1 \quad q>1
$$

by the dynamical variables $p, \xi, u$ of the $q$ deformed Heisenberg algebra

$$
p \xi-q \xi p=-i c u \quad q>1
$$

Let us briefly summarize the meaning of this algebra. It is based on a complex Hilbert space HS with orthonormal basis $\left\{|n\rangle_{\rho}^{\pi_{0}} \mid n \in Z, \rho \in S:=\right.$ $\left.\{1,-1\}, \pi_{0} \in R\right\}$. Following the approach of Wess it has turned out that this Hilbert space is directly related to a quantization of momentum and space via the parameter $q$, see [10]. We refer to this phenomenon by the expression 'Discrete Hilbert Space/HS'. As a consequence the spectrum of $p$ can be regarded as a lattice. Like in quantum

*e-mail: ruffing@appl-math.tu-muenchen.de 
mechanics the Hilbert space HS shall give all possible states of the free particle where

$$
{ }_{\rho}^{\pi_{0}}\langle n \mid m\rangle_{\sigma}^{\pi_{0}}=\delta_{n m} \delta_{\rho \sigma}, \quad \sum_{\rho \in S, j \in Z}|j\rangle_{\rho \rho}^{\pi_{0} \pi_{0}}\langle j|=1
$$

The parameter $\pi_{0}$ characterizes the chosen representation. In the special case $c=\left(q^{-1 / 2}-q^{-3 / 2}\right)$ matrix representations of $p, u, \xi$ are given by the action of the operators in the following sense

$$
\begin{gathered}
p|n\rangle_{\rho}^{\pi_{0}}=\rho \pi_{0} q^{n}|n\rangle_{\rho}^{\pi_{0}} \\
\xi|n\rangle_{\rho}^{\pi_{0}}=-i \rho^{-1} \pi_{0}^{-1} q^{-n} \\
\left(q^{1 / 2}|n-1\rangle_{\rho}^{\pi_{0}}-q^{-1 / 2}|n+1\rangle_{\rho}^{\pi_{0}}\right) \\
u|n\rangle_{\rho}^{\pi_{0}}=|n-1\rangle_{\rho}^{\pi_{0}} .
\end{gathered}
$$

$p$ is considered to be the momentum operator, $\xi$ is identified with the operator of space. $u$ turns out to be an operator which shifts one lattice point into its neighbour. The matrix representations yield a quantization of the spectrum belonging to the operator $\xi$. The quantization is related to the $q$ Fourier transform $[10,8]$. There are also a lot of possible representations for $p, u$ and $\xi$ in the context of $q$-hypergeometric functions [8,9]. In the following we choose the $q$-momentum representation which is given by the mapping

$$
p \rightarrow z, \xi \rightarrow D \text { ( } q \text {-differentiation }), u \rightarrow R
$$

Generalizing the quantum mechanical framework $z$ denotes the multiplication of a function with its variable. The operators $R, L, D$ are given by their action on a suitable function $f$ which has to be specified in the context. The actions are realized by shifting operators $R, L$ and the $q$-difference operator $D$

$$
\begin{gathered}
(R f)(z)=f(q z)(L f)(z)=f\left(q^{-1} z\right), \quad q>1 \\
(D f)(z):=\frac{f(q z)-f\left(q^{-1} z\right)}{q z-z / q}, \quad q>1
\end{gathered}
$$

From now on we restrict to $q>1$ following $[10,5,6]$. Clearly the limit $q \rightarrow 1$ turns $D$ into the differentiation of classical analysis. Questions concerning the limit were investigated in detail in [12] where also is shown that a suitable choice for the $q$-deformed Heisenberg algebra is

$$
p \xi-q \xi p=-i q^{3 / 2} u
$$

One finds that the representation allows a well defined limitation $q \rightarrow 1$. For remaining problems in the context of $q$-limitation see [12]. From the last equation follows the $q$-momentum representation for $p, u, \xi$. We will see that the expected representation of the algebra (1) in terms of the dynamical variables

$$
a=f(p, u, \xi) \quad a^{\dagger}=f^{\dagger}(p, u, \xi)
$$

yields a problem which in contrast to quantum mechanics is much more complicated. In [10] one can find a deeper investigation of questions concerning self-adjointness and essential self-adjointness of the involved operators $p, \xi, u$. We will apply these results briefly at the end of the fourth chapter. By the operation ' $t$ ' we denote the formal adjoint of the dynamical variables which is given by

$$
p^{\dagger}=p \quad \xi^{\dagger}=\xi \quad u^{\dagger}=u^{-1}
$$

i.e., for example

$$
\left(p|n\rangle_{\rho}^{\pi_{0}},|m\rangle_{\rho}^{\pi_{0}}\right)=\left(|n\rangle_{\rho}^{\pi_{0}}, p^{\dagger}|m\rangle_{\rho}^{\pi_{0}}\right) \quad m, n \in Z
$$

To deal with the mentioned non trivial situation in the $q$-case we will start in the second chapter from an observation which we call commutator symmetry or oscillator symmetry of Heisenberg algebras. Making use of it we can generalize the oscillator concept of quantum mechanics. The expression oscillator symmetry can be related to the corresponding dynamical symmetry (1). In the third chapter we will derive the $q$-Hermite functions which follow from the commutator 
symmetry. We will construct explicitly creators and annihilators in the $q$-momentum representation. We will also investigate the behaviour for $q \rightarrow 1$. Finally we transfer the result of the $q$ momentum representation to the situation in the abstract Hilbert space HS. Moreover we introduce an operator which can be regarded as a formal $q$-generalization of the quantum mechanical Hamiltonian for harmonic oscillators. For its interpretation in a $q$-deformed theory see $[5,6]$. Further investigations concerning the deeper physical meaning of this situation are presently prepared [3].

\section{OSCILLATOR SYMMETRIES OF HEISENBERG ALGEBRAS}

In quantum mechanics the ground state of the harmonic oscillator is given by the equation

$$
(p-i \xi)\left|\psi_{0}\right\rangle=0 \quad \text { or } \quad(p+i \xi)\left|\psi_{0}\right\rangle-2 p\left|\psi_{0}\right\rangle=0
$$

Applying the operator $(p+i \xi)$ repeatedly on this equation and making use of the Heisenberg algebra $p \xi-\xi p=i$ we obtain

$$
\begin{array}{r}
(p+i \xi)^{n+1}\left|\psi_{0}\right\rangle+\alpha_{n} p(p+i \xi)^{n}\left|\psi_{0}\right\rangle \\
+\beta_{n}(p+i \xi)^{n-1}\left|\psi_{0}\right\rangle=0
\end{array}
$$

where $n \in N$. The numbers $\alpha_{n}$ and $\beta_{n}$ are determined uniquely by the recursion.

The action of the operator $p+i \xi$ leaves the form of Eq. (15) invariant. It leads to the mentioned oscillator symmetry $a a^{\dagger}-a^{\dagger} a=1$. All that is well known from quantum mechanics so that we can be very brief. As the described situation admits a direct approach to the $a$ - $a^{\dagger}$-relations in quantum mechanics we want to generalize it in the $q$ deformed case.

Let us consider equations of the following type based on the $q$-Heisenberg algebra

$$
\begin{aligned}
\left(T+a u^{-1} \xi+b p\right)\left|\psi_{T}\right\rangle & =0 \\
\text { and } \quad(T+a u \xi+b p)\left|\psi_{T}\right\rangle & =0
\end{aligned}
$$

where $a$ and $b$ are arbitrary complex numbers. $T$ denotes an endomorphism of the Hilbert space HS and can be represented by its action on the eigenstates of $p$. Let $\operatorname{End}(\mathrm{HS})$ be the set of all endomorphisms in HS. Furthermore we want to restrict to all triples

$$
(T, a, b) \in(\operatorname{End}(\mathrm{HS}), C \times C)
$$

which allow solutions $\left|\psi_{T}\right\rangle$ of the Eq. (16) with a finite norm,

$$
\left|\psi_{T}\right\rangle=\sum_{n=-\infty}^{\infty} a_{n}|n\rangle_{+1}^{\pi_{0}}+\sum_{n=-\infty}^{\infty} b_{n}|n\rangle_{-1}^{\pi_{0}}\left(\pi_{0}=1\right)
$$

$$
\left\langle\psi_{T} \mid \psi_{T}\right\rangle=\sum_{n=-\infty}^{\infty}\left|a_{n}\right|^{2}+\left|b_{n}\right|^{2}<\infty
$$

Without loss of generality we will consider only the first equation of (16). Furthermore it turns out that the $q$-generalizations of recursion formula (15) require the following properties of $T$

1. $T$ fulfills the commutation relation $T p=q^{-2} p T$

2. For a given solution $\left|\psi_{T}\right\rangle$ of the equation $\left(T+a u^{-1} \xi+b p\right)\left|\psi_{T}\right\rangle=0$ the following elements of Hilbert space

$$
\left|\psi_{n}\right\rangle:=\left(T+a u^{-1} \xi\right)^{n}\left|\psi_{T}\right\rangle, \quad n=0,1,2, \ldots
$$

exist and have finite norm $\left\langle\psi_{n} \mid \psi_{n}\right\rangle<\infty$.

We call such a triple $(T, a, b) \in($ End(HS), $C \times C$ ) generating triple and $X:=T+a u^{-1} \xi$ the related transition operator as $T$ describes a mapping from $\left|\psi_{n}\right\rangle$ to $\left|\psi_{n+1}\right\rangle$ by

$$
\left(T+a u^{-1} \xi\right)\left|\psi_{n}\right\rangle=\left|\psi_{n+1}\right\rangle .
$$

It is worth noticing that any transition operator $X:=T+a u^{-1} \xi$ can be expressed in the form

$$
X=p u^{-2} h_{x} \quad h_{x}|n\rangle_{ \pm 1}^{\pi_{0}=1}=h_{x}\left( \pm q^{n}\right)|n\rangle_{ \pm 1}^{\pi_{0}=1} .
$$

where $h_{x}$ is a real valued function on the compact support $\sum:=\left\{ \pm q^{n} \mid n \in z\right\}, h_{x}: \sum \rightarrow R$. 
Let $(T, a, b)$ and $(S, c, d)$ be two generating sets, $X_{S}$ and $X_{T}$ the corresponding transition operators. We then have

$$
\begin{aligned}
\left(T+a u^{-1} \xi+b p\right)\left|\psi_{T}\right\rangle & =0, T p=q^{-2} p T, a, b \in C \\
\left(S+c u^{-1} \xi+d p\right)\left|\psi_{s}\right\rangle & =0, \quad S p=q^{-2} p S
\end{aligned}
$$

so that we immediately obtain

$$
\begin{aligned}
& \left(T+a u^{-1} \xi\right)^{n}\left|\psi_{T}\right\rangle=y_{n}(p)\left|\psi_{T}\right\rangle \\
& \quad \text { and }\left(n \in N_{0}\right)\left(S+c u^{-1} \xi\right)^{n}\left|\psi_{S}\right\rangle=y_{n}(p)\left|\psi_{S}\right\rangle .
\end{aligned}
$$

Note that the functions $y_{n}(p)$ are the same in both cases. They are easily determined to be polynomials in $p$. It can be shown that they fulfill recursion formulas of the following type

$$
y_{n+1}(p)+b q^{-2 n} p y_{n}(p)-i q^{-1}[n] a b y_{n-1}(p)=0,
$$

where

$$
[n]:=\frac{q^{-2 n}-1}{q^{-2}-1}, \quad n \in N
$$

In order to classify these polynomials let us consider the case $b=-1$ and $-i q^{-1} a b=1$ which plays the most important role in practice. Applying the left hand side of (21) on the state $|-2 m\rangle:=$ $|-2 m\rangle_{1}^{1}$ we obtain

$$
\begin{aligned}
& \left(y_{n+1}\left(q^{-2 m}\right)-q^{-2 n} q^{-2 m} y_{n}\left(q^{-2 m}\right)\right. \\
& \left.\quad+[n] y_{n-1}\left(q^{-2 m}\right)\right)|-2 m\rangle=0, \quad n \in N
\end{aligned}
$$

This holds for all eigenstates of $p$ which belong to even lattice points. These states span the even Hilbert space and therefore

$$
\begin{aligned}
& y_{n+1}\left(q^{-2 m}\right)-q^{-2 n} q^{-2 m} y_{n}\left(q^{-2 m}\right) \\
& \quad+[n] y_{n-1}\left(q^{-2 m}\right)=0
\end{aligned}
$$

The same argumentation is valid for the odd lattice points. It can be easily recognized that Eq. (22) yields polynomial functions $y_{n}:\left\{ \pm q^{2 m} \mid\right.$ $m \in Z\} \rightarrow R$. Equations of type (24) can be written in the form

$$
\begin{aligned}
& y_{n+1}\left(q^{-2 m}\right)-q^{-2 m} y_{n}\left(q^{-2 m}\right) \\
& \quad+\left(q^{-2}\right)^{-2 n+1}\left(1-q^{-2 n}\right) y_{n-1}\left(q^{-2 m}\right)=0, \quad n \in N
\end{aligned}
$$

From this equation we deduce the family of polynomials. The functions $y_{n}$ are said to be $q$ generalized discrete Hermite polynomials [4].

We expect these polynomials to characterize $q$ deformed oscillator relations which will be derived in the following section.

\section{3. $Q$-HERMITE FUNCTIONS AND $Q$-LADDER OPERATORS}

To generalize the situation of quantum theory let us fix $T$ and $i \alpha$ as elements of a generating set $(T, i \alpha, \beta), \alpha, \beta \in R$. We construct $q$-creation operators by the definition

$$
a^{\dagger}:=T+i \alpha u^{-1} \xi
$$

which can be rewritten in the form

$$
a^{\dagger}:=\beta p u^{-2} h(p)+i \alpha u^{-1} \xi
$$

In order to establish the expected $q$-oscillator relations $\alpha$ and $\beta$ have to be specified. In this context $a^{\dagger}$ is supposed to be a transition operator. The formal adjoint is given by

$$
a:=T^{\dagger}-i \bar{\alpha} \xi u
$$

We need to find a ground state $\left|\psi_{0}\right\rangle$ with finite norm so that the following equations hold

(1) $a\left|\psi_{0}\right\rangle=0$

(2) $a^{\dagger}\left|\psi_{0}\right\rangle=\gamma p\left|\psi_{0}\right\rangle, \gamma \in R$

(3) $\left|\psi_{n}\right\rangle=\gamma_{n}\left(a^{\dagger}\right)^{n}\left|\psi_{0}\right\rangle, \gamma_{n} \in R$

(4) $a^{\dagger} p=\left(T+i \alpha u^{-1} \xi\right) p=q^{-2} p\left(T+i \alpha u^{-1} \xi\right)+1=$ $q^{-2} p a^{\dagger}+1$

(5) $\left\langle\psi_{n} \mid \psi_{m}\right\rangle=\delta_{n m}$ 
Note that in quantum theory the equation of the ground state (1) implies the remaining relations (2)-(5). Making use of the momentum representation, given in the first chapter, Eqs. (2) and (3) read

$$
\begin{gathered}
\left(-q^{2} \alpha L_{q^{-2}} D_{q^{-2}}+\beta q^{2} L_{q^{-2}} f(x)\right) \psi_{0}(x)=0 \\
\left(\alpha D_{q^{-2}}+\beta f(x) R_{q^{-2}}\right) \psi_{0}(x)=\gamma x \psi_{0}(x)
\end{gathered}
$$

where $x \in\left\{ \pm q^{2 n}, \pm q^{2 n+1} \mid n \in Z\right\}$.

We show now that Eqs. (29), (30) have a common solution $\psi_{0}$ which is well defined for every real value $x$.

The first equation is equivalent to the equation $\left(\alpha D_{q^{-2}}+\beta f(x) R_{q^{-2}}\right) \psi_{0}(x)=0$. Substituting this last relation into (29) we receive

$$
D_{q^{-2}} \psi_{0}^{2}(x)=\frac{\gamma}{\alpha} x \psi_{0}^{2}(x)
$$

For the special choice $(\gamma / \alpha)=\left(q^{-2}-1\right)^{-1}$ a solution is given by the explicit expression

$$
\psi_{0}^{2}(x)=\left(i x ; q^{-2}\right)_{\infty}^{-1}\left(-i x ; q^{-2}\right)_{\infty}^{-1}
$$

Here we make use of the $q$-factorials defined by

$$
\begin{aligned}
\left(a ; q^{-2}\right)_{k} & :=\prod_{n=0}^{k-1}\left(1-a q^{-2 n}\right) \\
\left(a ; q^{-2}\right)_{\infty} & :=\lim _{k \rightarrow \infty}\left(a ; q^{-2}\right)_{k}
\end{aligned}
$$

Note that the parameter $\beta$ can be recognized as a part of the function $f$ and therefore may be omitted.

It is interesting to consider the special solutions $\psi_{0}^{2}$ belonging to the choice

$$
\begin{aligned}
& (\alpha, \gamma)=\left(1,\left(q^{-2}-1\right)^{-1}\right) \\
& \quad \text { and }(\gamma, \alpha)=\left(1, q^{-2}-1\right)
\end{aligned}
$$

from which we can learn most of the basic facts concerning $q$-generalized annihilators and creation operators.
With the choice $(\gamma, \alpha)=\left(1, q^{-2}-1\right)$ we derive from

$$
\left(\alpha D_{q^{-2}}+f R_{q^{-2}}\right) \psi_{0}(x)=\gamma x \psi_{0}(x)
$$

the recursion relation

$$
\begin{aligned}
& \left(\left(q^{-2}-1\right) D_{q^{-2}}+f R_{q^{-2}}\right)^{n+1} \psi_{0}(x) \\
& \quad-q^{-2 n} x\left(\left(q^{-2}-1\right) D_{q^{-2}}+f R_{q^{-2}}\right)^{n} \psi_{0}(x) \\
& +\left(1-q^{-2 n}\right)\left(\left(q^{-2}-1\right) D_{q^{-2}}\right. \\
& \left.\quad+f R_{q^{-2}}\right)^{n-1} \psi_{0}(x)=0
\end{aligned}
$$

Defining the polynomials

$$
h_{n}(x):=c_{n}\left(\left(q^{-2}-1\right) D_{q^{-2}}+f R_{q^{-2}}\right)^{n} \psi_{0}(x) / \psi_{0}(x),
$$

where $c_{n+1}:=q^{2 n} c_{n}, c_{0}=c_{1}=1$, we obtain from (36) the equation

$$
\begin{aligned}
& h_{n+1}(x)-x h_{n}(x) \\
& \quad+\left(q^{-2}\right)^{-2 n+1}\left(1-q^{-2 n}\right) h_{n-1}(x)=0 .
\end{aligned}
$$

By setting $x:=q^{-2 m}$ these functions reproduce exactly the polynomials $y_{n}$ given by (25). Note the important fact that these $q$-generalized Hermite functions are orthogonal with respect to the square of the ground state, $\psi_{0}^{2}$, i.e.,

$$
\begin{aligned}
& \sum_{k=-\infty}^{\infty}\left\{h_{n}\left(c q^{2 k}\right) h_{m}\left(c q^{2 k}\right)+h_{n}\left(-c q^{2 k}\right) h_{m}\left(-c q^{2 k}\right)\right\} \\
& \psi_{0}^{2}\left(c q^{2 k}\right) q^{2 k}=V_{n}(c) \delta_{n m}
\end{aligned}
$$

This equation holds for all positive numbers $c$ where $V_{n}(c)$ is a normalization factor. For abstract classification of the polynomial type see also [4].

The result obtained in this way is the expected $q$ version of the quantum mechanical orthogonality relation for continuous Hermite functions.

Next we consider the solution $\psi_{0}$ belonging to $(\alpha, \gamma)=\left(1,\left(q^{-2}-1\right)^{-1}\right)$. Equation (35) then reads

$$
\left(D_{q^{-2}}+f R_{q^{-2}}\right) \psi_{0}(x)=\left(q^{-2}-1\right)^{-1} x \psi_{0}(x)
$$


where $f(x):=\left(\left(\left(D_{q^{-2}} \psi_{0}\right)(x)\right) /\left(\psi_{0}(x)\right)\right)$. For simplicity of notation we introduce $\sigma:=\sqrt{\left(1-q^{-4}\right)}$ and obtain the following equation by applying the operator $R_{\sigma}$ on both sides of (40)

$$
\left(D_{q^{-2}}+\frac{\left(D_{q^{-2}} \psi^{0}\right)(x)}{\psi^{0}(x)}\right)=-\left(1+q^{-2}\right) x \psi^{0}(x),
$$

where

$$
\psi^{0}(x):=\left(R_{\sigma} \psi_{0}\right)(x)
$$

This rescaling procedure turns out to be suitable for the limitation $q \rightarrow 1$ as we will see later on. To proceed it is useful to introduce the operator

$$
A^{\dagger}:=\left(-D_{q^{-2}}-\frac{\left(D_{q^{-2}} \psi^{0}\right)(x)}{\psi^{0}(x)}\right) R_{q^{-2}}
$$

and its formal adjoint $A$. From (41) we obtain the recurrence relation

$$
\begin{gathered}
\left(A^{\dagger}\right)^{n+1} \psi^{0}-q^{-2 n}\left(1+q^{-2}\right) x\left(A^{\dagger}\right)^{n} \psi^{0} \\
+\left(1+q^{-2}\right)[n]\left(A^{\dagger}\right)^{n-1} \psi^{0}=0
\end{gathered}
$$

Defining the polynomials $H_{n}(x):=\left(\left(A^{\dagger}\right)^{n} \psi^{0}(x)\right) /$ $\psi^{0}(x)$ this relation may be written as

$$
\begin{gathered}
H_{n+1}(x)-\left(1+q^{-2}\right) q^{-2 n} x H_{n}(x) \\
\quad+\left(1+q^{-2}\right)[n] H_{n-1}(x)=0 .
\end{gathered}
$$

A straightforward calculation gives

$$
\begin{array}{r}
\left(1-q^{-2}\right)^{n / 2}\left(L_{\sigma} H_{n}\right)(x)\left(L_{\sigma} \psi_{0}\right)(x) \\
=\left(1+q^{-2}\right)^{n / 2} c_{n} h_{n}(x) \psi_{0}(x),
\end{array}
$$

where $c_{0}=c_{1}=1, c_{n+1}=q^{2 n} c_{n}, n \in N$. This leads almost immediately to the result (compare (39))

$$
\begin{aligned}
& \sum_{k=\infty}^{\infty}\left(H_{m} \psi^{0}\right)\left(c q^{2 k}\right)\left(H_{n} \psi^{0}\right)\left(c q^{2 k}\right) c q^{2 k} \\
& \quad+\sum_{k=\infty}^{\infty}\left(H_{m} \psi^{0}\right)\left(-c q^{2 k}\right)\left(H_{n} \psi^{0}\right)\left(-c q^{2 k}\right) c q^{2 k} \\
& \quad=V_{n}(c) \delta_{n m}
\end{aligned}
$$

Note that the functions $\left(H_{n} \psi^{0}\right)(x)$ show the mentioned phenomenon of scaling orthogonality which implies that the orthogonality of the functions does not depend on the positive parameter $c$ [4]. Compared to the case of classical Hermite polynomials this turns out to be an additional property of Hermite functions occuring in the $q$-generalized case.

Now having derived the orthogonality relation for the functions $H_{n} \psi^{0}$ let us discuss the basic properties of $A$ and $A^{\dagger}$. Formal calculation shows that

$$
A^{\dagger} x-q^{-2} x A^{\dagger}=1
$$

and therefore

$$
x A-q^{-2} A x=1 \Leftrightarrow A x=q^{2} x A-q^{2}
$$

Introducing the functions

$$
\begin{aligned}
\psi_{n}(x) & :=(-1)^{n} \gamma_{n}^{-1} H_{n}(x) / \psi^{0}(x) \\
\gamma_{n} & :=(-1)^{n}([n] !)^{1 / 2} \\
{[n] ! } & :=\prod_{j=1}^{n}[j]
\end{aligned}
$$

the action of the operator $A x$ on $\psi_{0}$ yields

$$
A x \psi_{0}=-q^{2} \psi_{0} \Leftrightarrow A \psi_{1}=-q^{2} \tau \psi_{0},
$$

as a consequence of putting $n=0$ in the recurrence relation (43). Therefore we know the induction statement

$$
A \psi_{n}=-q^{2} \tau[n]^{1 / 2} \psi_{n-1}
$$

for $n=0,1$. From the recurrence relation (44) we obtain

$$
\tau^{-1} \gamma_{n+1} \psi_{n+1}+q^{-2 n} x \gamma_{n} \psi_{n}+[n] \gamma_{n-1} \psi_{n-1}=0
$$

The operator $A$ acts on $q^{-2 n} x \gamma_{n} \psi_{n}$ as follows

$$
\begin{aligned}
& q^{-2 n} \gamma_{n} A x \psi_{n} \\
& \quad=q^{-2 n} \gamma_{n}\left(q^{2} x A-q^{2}\right) \psi_{n} \\
& \quad=q^{-2(n-1)} \gamma_{n} x(-1) q^{2} \tau[n]^{1 / 2} \psi_{n-1}
\end{aligned}
$$




$$
\begin{aligned}
& -q^{-2(n-1)} \gamma_{n} \psi_{n} \\
= & q^{-2(n-1)} x(-1)(-1)[n]^{1 / 2} \gamma_{n-1} q^{2} \tau[n]^{1 / 2} \psi_{n-1} \\
& -q^{-2(n-1)} \gamma_{n} \psi_{n} \\
= & q^{2} \tau[n] x q^{-2(n-1)} \gamma_{n-1} \psi_{n-1}-q^{-2(n-1)} \gamma_{n} \psi_{n} \\
= & -q^{2} \tau[n]\left(\tau^{-1} \gamma_{n} \psi_{n}+[n-1] \gamma_{n-2} \psi_{n-2}\right) \\
& -q^{-2(n-1)} \gamma_{n} \psi_{n} \\
= & -q^{2} \gamma_{n}\left([n]+\left(q^{-2}\right)^{n}\right) \psi_{n} \\
& -q^{2} \tau[n][n-1]_{q-2} \gamma_{n-2} \psi_{n-2} \\
= & -q^{2} \gamma_{n}[n+1]-q^{2} \tau[n][n-1] \gamma_{n-2} \psi_{n-2} .
\end{aligned}
$$

Furthermore we get

$$
\begin{aligned}
{[n] A \gamma_{n-1} \psi_{n-1} } & =-\gamma_{n-1} q^{2} \tau[n-1][n] \psi_{n-2} \\
& =\gamma_{n-2}[n-1][n] q^{2} \tau \psi_{n-2}
\end{aligned}
$$

Summarizing these computations we get the result

$$
\tau^{-1} A \gamma_{n+1} \psi_{n+1}-q^{2} \gamma_{n}[n+1] \psi_{n}=0
$$

which can be written as

$$
A \psi_{n+1}=\frac{\tau q^{2} \gamma_{n}}{\gamma_{n+1}[n+1]^{1 / 2}} \psi_{n}
$$

or

$$
A \psi_{n+1}=-q^{2} \tau[n+1]^{1 / 2} \psi_{n} .
$$

As we have seen (48) implies (51). This completes the proof of induction so that (48) holds for all $n \in N_{0}$.

The definition of the functions $\psi_{n}$ also yields

$$
A^{\dagger} \psi_{n}=[n]^{1 / 2} \psi_{n+1}
$$

This reveals a great similarity to the well known quantum mechanical generators and annhilators $a, a^{\dagger}$. In order to establish a complete analogy to the quantum mechanical $a-a^{\dagger}$-relation we perform a rescaling of (51) and (52) by putting

$$
\rho:=-q^{2}\left(1+q^{-2}\right) \quad \text { and } \quad \psi_{n} \rightarrow \psi^{n}:=\rho^{-n / 2} \psi_{n}
$$

Consequently we obtain the following result with two possible realizations of $A$ and $A^{\dagger}$

$$
\begin{gathered}
A^{\dagger} \psi^{n}= \pm \rho^{1 / 2}[n+1]^{1 / 2} \psi^{n+1} \\
A \psi^{n}= \pm \rho^{1 / 2}[n]^{1 / 2} \psi^{n-1}
\end{gathered}
$$

where $\rho^{1 / 2}:=i q\left(1+q^{-2}\right)^{1 / 2}$. The corresponding momentum representations read

$$
\begin{aligned}
& A^{\dagger}= \pm i q^{1 / 2}[2]^{-1}\left(D_{q^{-2}}+\left(D_{q^{-2}} \psi^{0} / \psi^{0}\right) R_{q^{-2}}\right) \\
& A= \pm i q^{1 / 2}[2]^{-1}\left(D_{q^{-2}}+\left(D_{q^{-2}} \psi^{0} / \psi^{0}\right) R_{q^{-2}}\right)^{+}
\end{aligned}
$$

From now on we choose the upper signs for $A$ and $A^{\dagger}$ in (56) and (57). We immediately obtain

$$
\begin{gathered}
A^{\dagger} \psi^{n}=[n+1]^{1 / 2} \psi^{n+1} \\
A \psi^{n}=[n]^{1 / 2} \psi^{n-1}
\end{gathered}
$$

This provides the existence of an orthonormal set $\left\{\psi^{n} \mid n \in N_{0}\right\}$ which can be easily verified by calculating the scalar products by means of (58) (59).

All functions $\left\{\psi^{n}\right\}$ are orthogonal up to a constant factor,

$$
\begin{aligned}
& \sum_{k=-\infty}^{\infty}\left(\psi^{n}\left(c q^{2 k}\right) \psi^{m}\left(c q^{2 k}\right)\right. \\
& \left.\quad+\psi^{n}\left(-c q^{2 k}\right) \psi^{m}\left(-c q^{2 k}\right)\right) q^{2 k}=\delta_{n m} \text { const. }
\end{aligned}
$$

The same argumentation holds for the above mentioned second solution $(\alpha, \gamma)=\left(\left(q^{-2}-1\right), 1\right)$.

It can easily be verified that (58) and (59) yield

$$
A A^{\dagger} \psi^{n}-q^{-2} A^{\dagger} A \psi^{n}=\psi^{n} \quad\left(n \in N_{0}\right)
$$

This relation is directly related to the symmetry $S U_{q}(1,1)$. 


\section{REPRESENTATION OF THE SYMMETRY $S U_{Q}(1,1)$}

Let us now apply the differential representation of the first chapter. Without loss of generality we choose $\pi_{0}=1$. We denote by $|n\rangle$ the eigenvectors of momentum which belong to the given $\pi_{0}=1$ and we define $\gamma:=-i[2]_{q^{-2}}^{1 / 2}$.

In the above introduced abstract Hilbert space HS the operator $a^{\dagger}$ is given by

$$
a^{\dagger}:=\gamma\left(q^{-2} p h u^{-2}+q^{-3}(\xi p-q p \xi) \xi\right)
$$

where

$$
h(p):=-p^{-1}\left(\psi^{0}(p)\right)^{-1} D_{q^{-2}} \psi^{0}(p) .
$$

Its momentum representation reads

$$
\operatorname{Repr}_{p}\left(a^{\dagger}\right)=-q^{-1} \gamma\left(D_{q^{-2}}+\left(\psi^{0}\right)^{-1}\left(D_{q^{-2}} \psi^{0}\right) R_{q^{-2}}\right) .
$$

An evaluation of $\left(\psi^{0}\right)^{-1}\left(D_{q^{-2}} \psi^{0}\right)$ yields the following result for $h$

$$
h(p)=\left(q^{-2}-1\right) p^{-2}\left(1-\left(1+\left(1-q^{-4}\right) p^{2}\right)^{+1 / 2}\right) .
$$

Performing two levels of expansion we get

$$
h(p)=\frac{1}{2}\left(1+q^{-2}\right)-\frac{1}{8}\left(1+q^{-2}\right)\left(1-q^{-4}\right) p^{2}+\cdots
$$

for all vectors $|n\rangle$ for which $p^{2}|n\rangle=q^{-2 n}|n\rangle$ and $q^{-2 n}\left(1-q^{-4}\right)<1$.

For a given $q$ we denote the number of all vectors satisfying the last condition by $N_{q}$. It is evident that $N_{q}$ is finite. One can show that $N_{q} \rightarrow \infty$ as $q \rightarrow 1$. This means $h(p) \rightarrow 1$ as $q \rightarrow 1$.

The last observation leads immediately to the general result that $\lim _{q \rightarrow 1} \psi^{0}(x)=e^{-(1 / 2) x^{2}}$ where the limit exists pointwise. This can also be seen by considering the general eigenvalue equation $p^{2}|n\rangle_{\pi_{0}}=\pi_{0}^{2} q^{2 n}|n\rangle_{\pi_{0}}$ and by taking into account that $R$ naturally is generated by the superposition of the open sets consisting of the spectral points $\pi_{0}^{2} q^{2 n}$ which belong to $\pi_{0}\left(\pi_{0}\right.$ varying in $R$ ).

We recall that by the calculations of the third and fourth chapter the formal adjoint of $a$ is given by

$$
a=-\gamma\left(p u^{2} h+q^{-3} \xi(p \xi-q \xi p)\right)
$$

The properties of the function $h$ show that the limit $q \rightarrow 1$ turns $a$ and $a^{\dagger}$ into

$$
a=i \frac{(p-i \xi)}{\sqrt{2}}, \quad a^{\dagger}=-i \frac{(p+i \xi)}{\sqrt{2}}
$$

where $p \xi-\xi p=i$. Thus we obtain the well known creators and annihilators of quantum mechanics.

Making use of the obtained results we define the following states of the given Hilbert space

$$
\begin{aligned}
\left|\psi_{m}^{x}\right\rangle:= & \left(1-q^{-2}\right)^{1 / 2} \sum_{n=-\infty}^{\infty} q^{n} \psi^{m}\left(q^{2 n}\right)|2 n\rangle \\
& +\sum_{n=-\infty}^{\infty} q^{n} \psi^{m}\left(-q^{2 n}\right)|-2 n\rangle \\
\left|\psi_{m}^{y}\right\rangle:= & \left(1-q^{-2}\right)^{1 / 2} \sum_{n=-\infty}^{\infty} q^{n+1 / 2} \psi^{m}\left(q^{2 n+1}\right)|2 n+1\rangle \\
& +\sum_{n=-\infty}^{\infty} q^{n+1 / 2} \psi^{m}\left(-q^{2 n+1}\right)|-2 n-1\rangle
\end{aligned}
$$

where $m \in N_{0}$.

Furthermore we find

$$
\begin{gathered}
a^{\dagger}\left|\psi_{n}^{x}\right\rangle=[n+1]^{1 / 2}\left|\psi_{n+1}^{x}\right\rangle \\
a^{\dagger}\left|\psi_{n}^{y}\right\rangle=[n+1]^{1 / 2}\left|\psi_{n+1}^{y}\right\rangle \\
a\left|\psi_{n}^{x}\right\rangle=[n]^{1 / 2}\left|\psi_{n-1}^{x}\right\rangle \quad a\left|\psi_{n}^{y}\right\rangle=[n]^{1 / 2}\left|\psi_{n-1}^{y}\right\rangle
\end{gathered}
$$

Moreover this leads to a $q$-generalization of the well known quantum mechanical relations between $a$ and $a^{\dagger}$

$$
\left(a a^{\dagger}-q^{-2} a^{\dagger} a\right)\left|\psi_{n}^{x, y}\right\rangle=\left|\psi_{n}^{x, y}\right\rangle \quad\left(n \in N_{0}\right) .
$$


As a consequence we receive the following eigenvalue equations

$$
\begin{gathered}
a^{\dagger} a\left|\psi_{n}^{x}\right\rangle=[n]\left|\psi_{n}^{x}\right\rangle \quad a^{\dagger} a\left|\psi_{n}^{y}\right\rangle=[n]\left|\psi_{n}^{y}\right\rangle \\
\left\langle\psi_{n}^{x} \mid \psi_{m}^{x}\right\rangle=\delta_{n m}\left\langle\psi_{n}^{y} \mid \psi_{m}^{y}\right\rangle=\delta_{n m}\left\langle\psi_{n}^{x} \mid \psi_{m}^{y}\right\rangle=0
\end{gathered}
$$

The momentum representation of the eigenstates becomes the eigenfunctions of the quantum mechanical harmonic oscillator as $q \rightarrow 1$.

We can show that $a$ is dense in Hilbert space, i.e., the domain $D(a) \subseteq$ HS is dense in HS. For a general framework see also [17]. Let us define the set

$$
\begin{aligned}
D^{*}:= & \{|\psi\rangle \in \operatorname{HS}|\exists| \psi\rangle^{*} \in \operatorname{HS} \forall|\varphi\rangle \\
& \left.\in D\left(a^{*}\right):(a|\varphi\rangle,|\psi\rangle)=\left(|\varphi\rangle,|\psi\rangle^{*}\right)\right\},
\end{aligned}
$$

i.e., the operator $a^{*}: D^{*} \rightarrow \mathrm{HS}, a^{*}|\psi\rangle:=|\psi\rangle^{*}$ for all $|\psi\rangle \in D^{*}$ is the adjoint of $a$. The operator $a^{*}$ is always closed and one can show that all eigenvectors of the momentum operator $p$ are in $D(a)$. Therefore $a^{*}$ is densely defined in HS. As a consequence [13] the operator

$$
H=a^{* *} a^{*}
$$

is self-adjoint. As $a^{*}$ is dense and closed we find

$$
a^{*}\left|\psi_{n}^{x}\right\rangle=a^{\dagger}\left|\psi_{n}^{x}\right\rangle \quad a^{*}\left|\psi_{n}^{y}\right\rangle=a^{\dagger}\left|\psi_{n}^{y}\right\rangle
$$

Omitting the symbols $x$ and $y$ we receive

$$
\left(a^{*}\left|\psi_{n}\right\rangle, a^{*}\left|\psi_{n}\right\rangle\right)=[n+1]<\infty
$$

This means that the vector $\left|\varphi_{m}\right\rangle:=a^{*}\left|\psi_{m}\right\rangle$ is a well defined element of the Hilbert space, i.e., $\left|\varphi_{m}\right\rangle=\left|\psi_{m+1}\right\rangle$. We define $a^{* *}\left|\varphi_{m}\right\rangle$ by the relation

$$
\left(a^{*}\left|\psi_{n}\right\rangle,\left|\varphi_{m}\right\rangle\right)=:\left(\left|\psi_{n}\right\rangle, a^{* *}\left|\varphi_{m}\right\rangle\right)=\delta_{m n}
$$

By the definitions $\left|\varphi_{0}\right\rangle:=\left|\psi_{0}\right\rangle$ and $a^{* *}\left|\psi_{0}\right\rangle:=0 \in C$ the action of the linear form $a^{* *}$ on the basis of HS is completely determined. As an easy consequence we find $\left|\varphi_{n}\right\rangle \in D\left(a^{* *}\right)$. This means

$$
\begin{aligned}
\left(a^{*}\left|\psi_{n}\right\rangle, a^{*}\left|\psi_{m}\right\rangle\right) & =\left(a^{*}\left|\psi_{n}\right\rangle,\left|\varphi_{m}\right\rangle\right) \\
& =\left(\left|\psi_{n}\right\rangle, a^{* *}\left|\varphi_{m}\right\rangle\right) \\
& =\left(\left|\psi_{n}\right\rangle, a^{* *} a^{*}\left|\psi_{m}\right\rangle\right) \\
& =\delta_{m n}[n+1]
\end{aligned}
$$

Let $H_{\mid}$denote the restriction of $H$ on the subspace of HS which consists of all finite linear combinations $\sum_{n=0}^{m \in N} a_{n}\left|\psi_{n}\right\rangle$. Obviously $a^{\dagger} a$ coincides with $H_{\mid}$.

\section{CONCLUSIONS}

The operator $H:=a^{\dagger} a$ is an oscillator like expression. For details of its physical meaning see [3]. In the $q$-deformed case the interpretation in the context of $S U_{q}(1,1)$ symmetry is investigated [5-7].

The action of $H$ on the $q$-Hermite basis elements of HS is given by

$$
H\left|\psi_{n}^{x}\right\rangle=[n]\left|\psi_{n}^{x}\right\rangle, \quad H\left|\psi_{n}^{y}\right\rangle=[n]\left|\psi_{n}^{y}\right\rangle
$$

Let us finally give the representation of $p$ and $\xi$ by operators $a$ and $a^{\dagger}$ as a generalization of the quantum mechanical situation

$$
\begin{aligned}
& p=-q^{-1 / 2} \gamma \sum_{n=0}^{\infty}\left(1-q^{-2}\right)^{n}\left(a^{\dagger} a\right)^{n}\left(q a^{\dagger}-a\right) \\
& \xi=i q^{3 / 2} \gamma\left(\sum_{n=0}^{\infty}(-1)^{n} q^{-n} u^{-2 n+1}\right)\left(a u^{-2}+u^{2} a^{\dagger}\right) .
\end{aligned}
$$

One can easily see that these operators are invariant under the formal adjoint $\dagger$ which is necessary for a consistent formulation of $q$ generalized quantum mechanics. They become the well known objects momentum $p$ and space $\xi(p \xi-\xi p=i)$ of quantum mechanics as $q \rightarrow 1$.

As a concluding result we have represented the $S U_{q}(1,1)$ symmetry algebra $a a^{\dagger}-q^{-2} a^{\dagger} a=1$ in terms of the dynamical variables $p, u, \xi$. The 
solution of this nontrivial problem reveals a further rich structure in the context of quantum symmetric quantum mechanics. More work has to be done on this area.

\section{Acknowledgements}

The author wishes to thank Julius Wess, Peter Schupp and their theory groups for very fruitful discussions and suggestions especially during the Enrico Fermi Summer School in Varenna and T. H. Koornwinder for communication.

\section{References}

[1] Macfarlane, A. (1989). J. Phys., A22, 4581.

[2] Biedenharn, L. (1989). J. Phys., A22, L873.

[3] Ruffing, A. (1996). Doctorate Thesis, LMU München.

[4] Koekoek, R. and Swarttouw, R. F., Report of the Faculty of Technical Mathematics and Informatics, No. 94-05, ISSN 0922-5641.

[5] Wess, J., Private Communications.
[6] Wess, J. et al., Dynamical Symmetries in $q$-deformed Quantum Mechanics MPI-PhT/95-1, GK-MP 9502/17, $q$-alg/9502007.

[7] Wess, J. et al., A $q$-Deformation of the Harmonic Oscillator, MPI-PhT/96-26.

[8] Jochen Schwenk, $q$-deformed Fourier Theory, MPI-PhT/ 94-36.

[9] Koornwinder, T. H. and Swarttouw, R. F. (1992). On $q$-analogues of the Fourier and Hankel transforms, Trans. AMS 333, 1, 445.

[10] Wess, J. et al., Representations of a $q$-deformed Heisenberg algebra, MPI-Ph/93-45, LMU-TPW 93-17 to appear in Z. Phys. C.

[11] Hurwitz-Courant, Funktionentheorie, Springer Verlag, Berlin-Göttingen-Heidelberg-New York, 1964.

[12] Fichtmüller, M. (1994). Diploma Thesis 'Der Grenzübergang $q \rightarrow 1$ der $q$-Fouriertransformation', LMU München.

[13] Riesz, F. and Sz-Nagy, B., Vorlesungen über Funktionalanalysis, VEB Deutscher Verlag der Wissenschaften, Berlin, 1956.

[14] Kempf, A., Private Communications.

[15] Wess, J. et al. (1992). A q-deformed quantum mechanical toy model, Phys. Lett., B291, 273.

[16] Hebecker, A. and Weich, W. (1992). Free particle in $q$-deformed Configuration Space, Lett. Math. Phys., 26, 245.

[17] Achieser, N. I. and Glasmann, I. M. (1954). Theorie der linearen Operatoren im Hilbertraum, Akademie-Verlag, Berlin. 


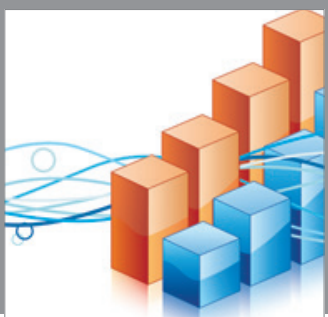

Advances in

Operations Research

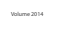

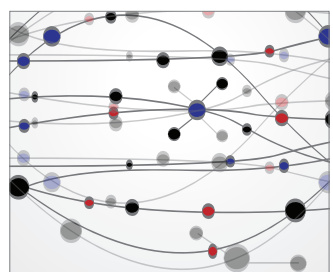

\section{The Scientific} World Journal
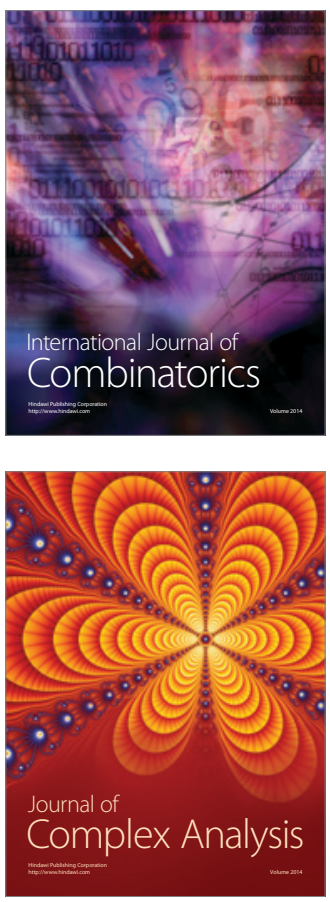

International Journal of

Mathematics and

Mathematical

Sciences
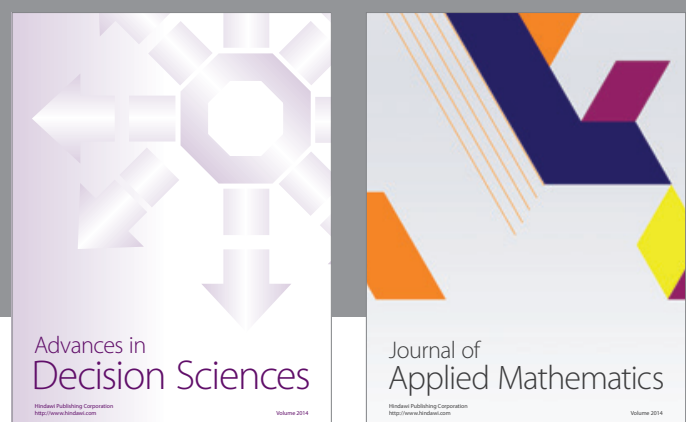

Journal of

Applied Mathematics
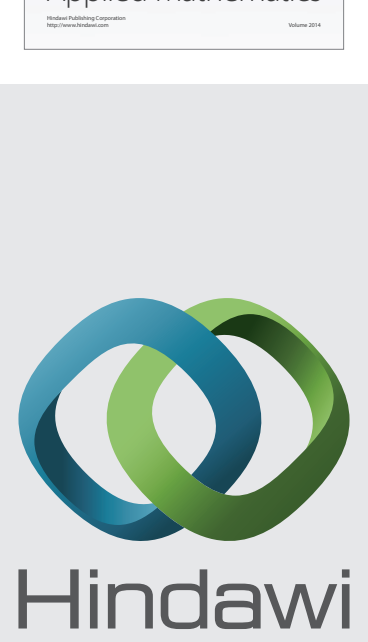

Submit your manuscripts at http://www.hindawi.com
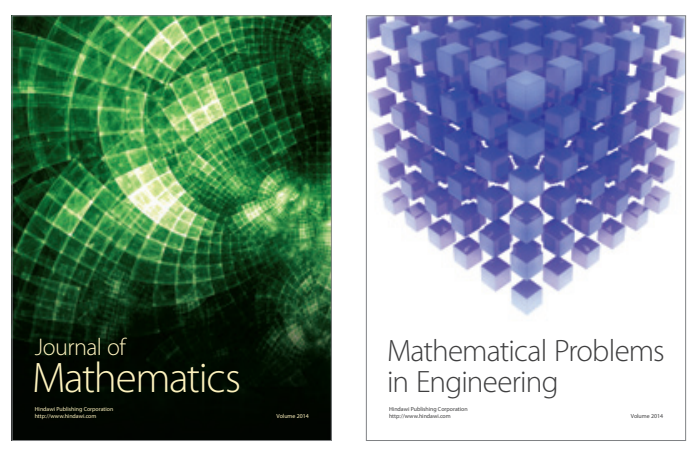

Mathematical Problems in Engineering
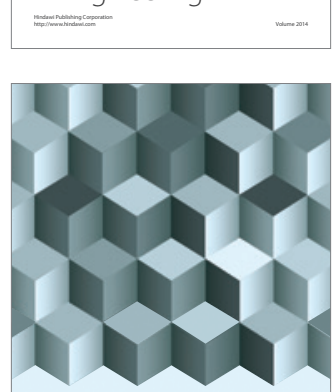

Journal of

Function Spaces
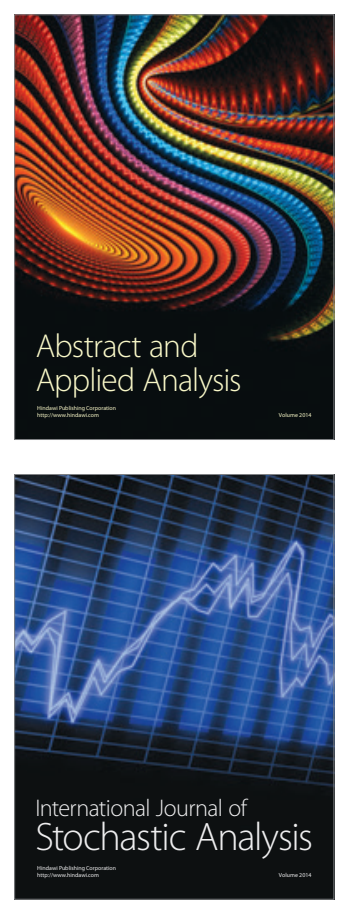

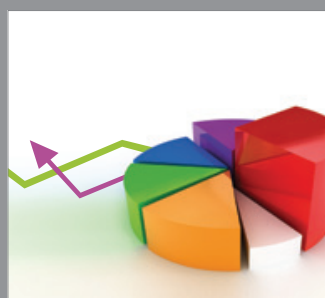

ournal of

Probability and Statistics

Promensencen
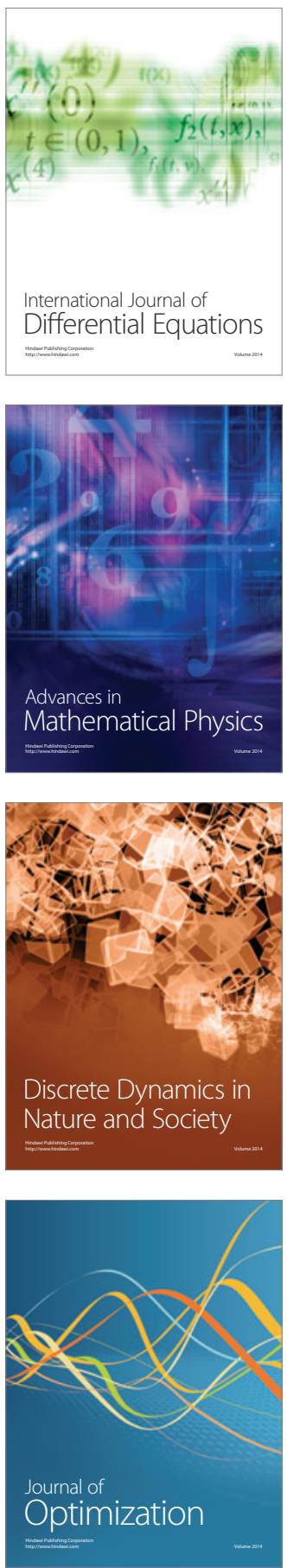